\title{
Is BERT Really Robust? A Strong Baseline for Natural Language Attack on Text Classification and Entailment
}

\author{
Di Jin, ${ }^{1 *}$ Zhijing Jin, ${ }^{2 *}$ Joey Tianyi Zhou, ${ }^{3}$ Peter Szolovits ${ }^{1}$ \\ ${ }^{1}$ Computer Science \& Artificial Intelligence Laboratory, MIT \\ ${ }^{2}$ University of Hong Kong \\ ${ }^{3} \mathrm{~A} *$ STAR, Singapore \\ \{jindi15,psz\}@mit.edu, zhijing.jin@connect.hku.hk, zhouty@ihpc.a-star.edu.sg
}

\begin{abstract}
Machine learning algorithms are often vulnerable to adversarial examples that have imperceptible alterations from the original counterparts but can fool the state-of-the-art models. It is helpful to evaluate or even improve the robustness of these models by exposing the maliciously crafted adversarial examples. In this paper, we present TEXTFOOLER, a simple but strong baseline to generate adversarial text. By applying it to two fundamental natural language tasks, text classification and textual entailment, we successfully attacked three target models, including the powerful pre-trained BERT, and the widely used convolutional and recurrent neural networks. We demonstrate three advantages of this framework: (1) effective-it outperforms previous attacks by success rate and perturbation rate, (2) utility-preserving-it preserves semantic content, grammaticality, and correct types classified by humans, and (3) efficient-it generates adversarial text with computational complexity linear to the text length. ${ }^{1}$
\end{abstract}

\section{Introduction}

In the last decade, Machine Learning (ML) models have achieved remarkable success in various tasks such as classification, regression and decision making. However, recently they have been found vulnerable to adversarial examples that are legitimate inputs altered by small and often imperceptible perturbations (Kurakin, Goodfellow, and Bengio 2016a; 2016b; Papernot et al. 2017; Zhao, Dua, and Singh 2017). These carefully curated examples are correctly classified by a human observer but can fool a target model, raising serious concerns regarding the security and integrity of existing ML algorithms. On the other hand, it is showed that robustness and generalization of ML models can be improved by crafting high-quality adversaries and including them in the training data (Goodfellow, Shlens, and Szegedy 2015).

While existing works on adversarial examples have obtained success in the image and speech domains (Szegedy et al. 2013; Carlini and Wagner 2018), it is still challenging to

\footnotetext{
*Equal Contribution. Order determined by swapping that in the previous paper at https://arxiv.org/abs/1901.11333.

Copyright (C) 2020, Association for the Advancement of Artificial Intelligence (www.aaai.org). All rights reserved.

${ }^{1}$ The code, pre-trained target models, and test examples are available at https://github.com/jind11/TextFooler.
}

deal with text data due to its discrete nature. Formally, besides the ability to fool the target models, outputs of a natural language attacking system should also meet three key utilitypreserving properties: (1) human prediction consistencyprediction by humans should remain unchanged, (2) semantic similarity-the crafted example should bear the same meaning as the source, as judged by humans, and (3) language fluency-generated examples should look natural and grammatical. Previous works barely conform to all three requirements. For example, methods such as word misspelling ( $\mathrm{Li}$ et al. 2018; Gao et al. 2018), single-word erasure (Li, Monroe, and Jurafsky 2016), and phrase insertion and removal (Liang et al. 2017) result in unnatural sentences. Moreover, there is almost no work that attacks the newly-risen BERT model on text classification.

In this work, we present TEXTFOOLER, a simple but strong baseline for natural language attack in the blackbox setting, a common case where no model architecture or parameters are accessible. We design a more comprehensive paradigm to create both semantically and syntactically similar adversarial examples that meet the aforementioned three desiderata. Specifically, we first identify the important words for the target model and then prioritize to replace them with the most semantically similar and grammatically correct words until the prediction is altered. We successfully applied this framework to attack three state-of-the-art models in five text classification tasks and two textual entailment tasks, respectively. On the adversarial examples, we can reduce the accuracy of almost all target models in all tasks to below $10 \%$ with only less than $20 \%$ of the original words perturbed. In addition, we validate that the generated examples are (1) correctly classified by human evaluators, (2) semantically similar to the original text, and (3) grammatically acceptable by human judges.

Our main contributions are summarized as follows:

- We propose a simple but strong baseline, TEXTFoOLER, to quickly generate high-profile utility-preserving adversarial examples that force the target models to make wrong predictions under the black-box setting.

- We evaluate TEXTFooler on three state-of-the-art deep learning models over five popular text classification tasks and two textual entailment tasks, and it achieved the state- 
of-the-art attack success rate and perturbation rate.

- We propose a comprehensive four-way automatic and three-way human evaluation of language adversarial attacks to evaluate the effectiveness, efficiency, and utilitypreserving properties of our system.

- We open-source the code, pre-trained target models, and test samples for the convenience of future benchmarking.

\section{Method}

\section{Problem Formulation}

Given a corpus of $N$ sentences $\mathcal{X}=\left\{X_{1}, X_{2}, \ldots, X_{N}\right\}$, and a corresponding set of $N$ labels $\mathcal{Y}=\left\{Y_{1}, Y_{2}, \ldots, Y_{N}\right\}$, we have a pre-trained model $F: \mathcal{X} \rightarrow \mathcal{Y}$, which maps the input text space $\mathcal{X}$ to the label space $\mathcal{Y}$.

For a sentence $X \in \mathcal{X}$, a valid adversarial example $X_{a d v}$ should conform to the following requirements:

$$
F\left(X_{a d v}\right) \neq F(X) \text {, and } \operatorname{Sim}\left(X_{a d v}, X\right) \geq \epsilon,
$$

where $\operatorname{Sim}: \mathcal{X} \times \mathcal{X} \rightarrow(0,1)$ is a similarity function and $\epsilon$ is the minimum similarity between the original and adversarial examples. In the natural language domain, Sim is often a semantic and syntactic similarity function.

\section{Threat Model}

Under the black-box setting, the attacker is not aware of the model architecture, parameters, or training data. It can only query the target model with supplied inputs, getting as results the predictions and corresponding confidence scores.

The proposed approach for adversarial text generation is shown in Algorithm 1, and consists of the two main steps:

Step 1: Word Importance Ranking (line 1-6) Given a sentence of $n$ words $X=\left\{w_{1}, w_{2}, \ldots, w_{n}\right\}$, we observe that only some key words act as influential signals for the prediction model $F$, echoing with the discovery of (Niven and Kao 2019) that BERT attends to the statistical cues of some words. Therefore, we create a selection mechanism to choose the words that most significantly influence the final prediction results. Using this selection process, we minimize the alterations, and thus maintain the semantic similarity as much as possible.

Note that the selection of important words is trivial in a white-box scenario, as it can be easily solved by inspecting the gradients of the model $F$, while most other words are irrelevant. However, under the more common black-box set up in our paper, the model gradients are unavailable. Therefore, we create a selection mechanism as follows. We use the score $I_{w_{i}}$ to measure the influence of a word $w_{i} \in X$ towards the classification result $F(X)=Y$. We denote the sentence after deleting the word $w_{i}$ as $X_{\backslash w_{i}}=X \backslash\left\{w_{i}\right\}=$ $\left\{w_{1}, \ldots, w_{i-1}, w_{i+1}, \ldots w_{n}\right\}$, and use $F_{Y}(\cdot)$ to represent the prediction score for the $Y$ label.

The importance score $I_{w_{i}}$ is therefore calculated as the prediction change before and after deleting the word $w_{i}$, which is formally defined as follows,

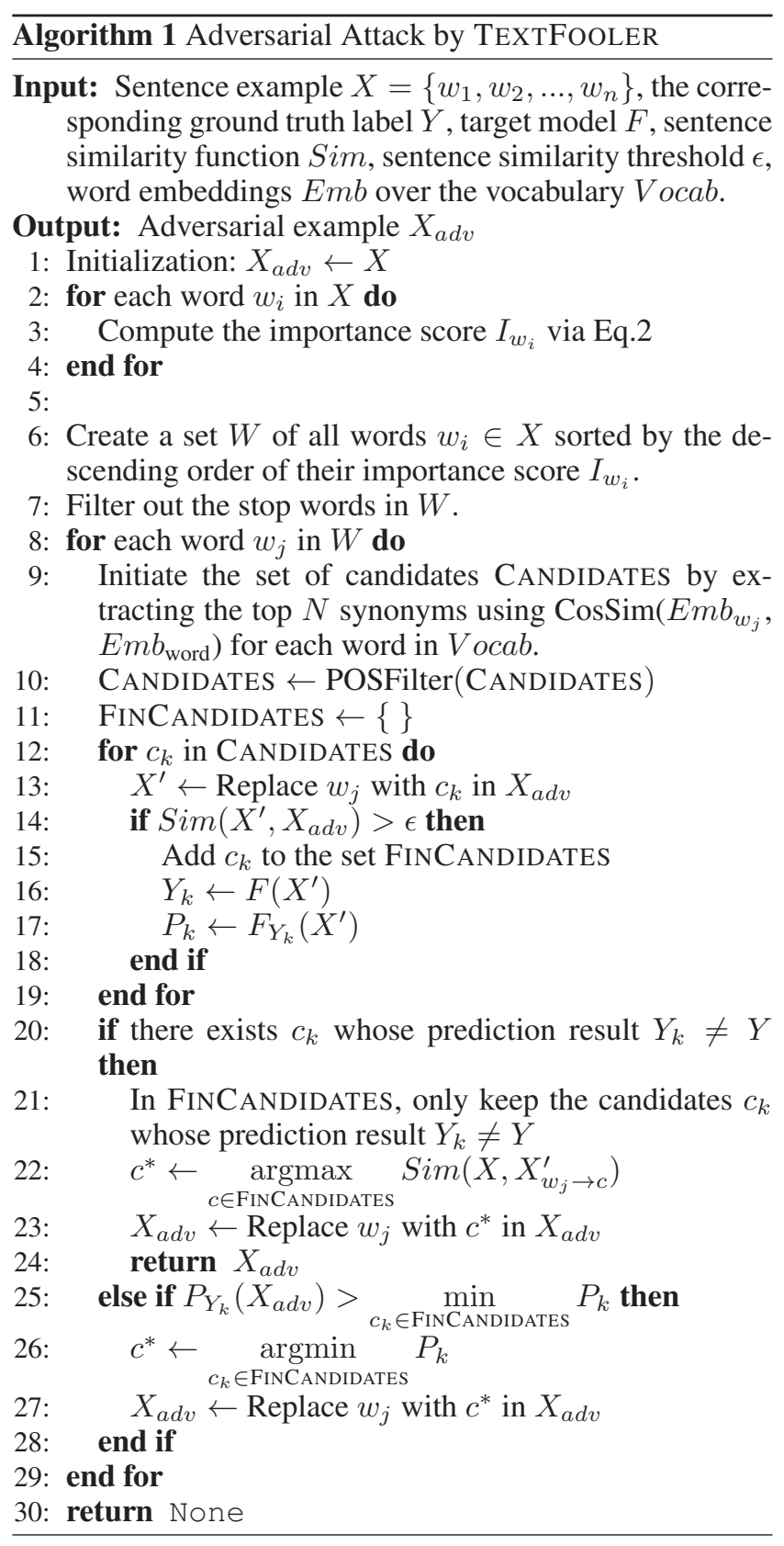

After ranking the words by their importance score, we further filter out stop words derived from $\mathrm{NLTK}^{2}$ and $\mathrm{spaCy}^{3}$ libraries such as "the", "when", and "none". This simple step of filtering is important to avoid grammar destruction.

\footnotetext{
${ }^{2}$ https://www.nltk.org/

${ }^{3}$ https://spacy.io/
} 
Step 2: Word Transformer (line 7-30) For a given word $w_{i} \in X$ with a high importance score obtained in Step 1, we need to design a word replacement mechanism. A suitable replacement word needs to fulfill the following criteria: it should (1) have similar semantic meaning with the original one, (2) fit within the surrounding context, and (3) force the target model to make wrong predictions. In order to select replacement words that meet such criteria, we propose the following workflow.

Synonym Extraction: We gather a candidate set CANDIDATES for all possible replacements of the selected word $w_{i}$. CANDIDATES is initiated with $N$ closest synonyms according to the cosine similarity between $w_{i}$ and every other word in the vocabulary.

To represent the words, we use word embeddings from (Mrkšić et al. 2016). These word vectors are specially curated for finding synonyms, as they achieve the state-of-theart performance on SimLex-999, a dataset designed to measure how well different models judge semantic similarity between words (Hill, Reichart, and Korhonen 2015).

Using this set of embedding vectors, we identify top $N$ synonyms whose cosine similarity with $w$ are greater than $\delta$. Note that enlarging $N$ or lowering $\delta$ would both generate more diverse synonym candidates; however, the semantic similarity between the adversary and the original sentence would decrease. In our experiments, empirically setting $N$ to be 50 and $\delta$ to be 0.7 strikes a balance between diversity and semantic similarity control.

POS Checking: In the set CANDIDATES of the word $w_{i}$, we only keep the ones with the same part-of-speech (POS) ${ }^{4}$ as $w_{i}$. This step is to assure that the grammar of the text is mostly maintained (line 10 in Algorithm 1).

Semantic Similarity Checking: For each remaining word $c \in$ CANDidates, we substitute it for $w_{i}$ in the sentence $X$, and obtain the adversarial example $X_{a d v}=$ $\left\{w_{1}, \ldots, w_{i-1}, c, w_{i+1}, \ldots, w_{n}\right\}$. We use the target model $F$ to compute the corresponding prediction scores $F\left(X_{a d v}\right)$. We also calculate the sentence semantic similarity between the source $X$ and adversarial counterpart $X_{a d v}$. Specifically, we use Universal Sentence Encoder (USE) (Cer et al. 2018) to encode the two sentences into high dimensional vectors and use their cosine similarity score as an approximation of semantic similarity. The words resulting in similarity scores above a preset threshold $\epsilon$ are placed into the final candidate pool FinCANDIDATES (line 11-19 in Algorithm 1).

Finalization of Adversarial Examples: In the final candidate pool FINCANDIDATES, if there exists any candidate that can already alter the prediction of the target model, then we select the word with the highest semantic similarity score among these winning candidates. But if not, then we select the word with the least confidence score of label $y$ as the best replacement word for $w_{i}$, and repeat Step 2 to transform the next selected word (line 20-30 in Algorithm 1).

Overall, the algorithm first uses Step 1 to rank the words by their importance scores, and then repeats Step 2 to find replacements for each word in the sentence $X$ until the pre-

\footnotetext{
${ }^{4} \mathrm{We}$ used the off-the-shelf spaCy tagger, available at https://spacy.io/api/tagger
}

diction of the target model is altered.

\section{Experiments}

\section{Tasks}

We study the effectiveness of our adversarial attack on two important NLP tasks, text classification and textual entailment. The dataset statistics are summarized in Table 1. Following the practice by Alzantot et al. (2018), we evaluate our algorithm on a set of 1,000 examples randomly selected from the test set.

\begin{tabular}{c|l|ccc}
\hline Task & Dataset & Train & Test & Avg Len \\
\hline \multirow{5}{*}{ Classification } & AG's News & $30 \mathrm{~K}$ & $1.9 \mathrm{~K}$ & 43 \\
& Fake News & $18.8 \mathrm{~K}$ & $2 \mathrm{~K}$ & 885 \\
& MR & $9 \mathrm{~K}$ & $1 \mathrm{~K}$ & 20 \\
& IMDB & $25 \mathrm{~K}$ & $25 \mathrm{~K}$ & 215 \\
& Yelp & $560 \mathrm{~K}$ & $38 \mathrm{~K}$ & 152 \\
\hline \multirow{2}{*}{ Entailment } & SNLI & $570 \mathrm{~K}$ & $3 \mathrm{~K}$ & 8 \\
& MultiNLI & $433 \mathrm{~K}$ & $10 \mathrm{~K}$ & 11 \\
\hline
\end{tabular}

Table 1: Overview of the datasets.

Text Classification To study the robustness of our model, we use text classification datasets with various properties, including news topic classification, fake news detection, and sentence- and document-level sentiment analysis, with average text length ranging from tens to hundreds of words.

- AG's News (AG): Sentence-level classification with regard to four news topics: World, Sports, Business, and Science/Technology. Following the practice of Zhang, Zhao, and LeCun (2015), we concatenate the title and description fields for each news article.

- Fake News Detection (Fake): Document-level classification on whether a news article is fake or not. The dataset comes from the Kaggle Fake News Challenge. ${ }^{5}$

- MR: Sentence-level sentiment classification on positive and negative movie reviews (Pang and Lee 2005). We use $90 \%$ of the data as the training set and $10 \%$ as the test set, following the practice in ( $\mathrm{Li}$ et al. 2018).

- IMDB: Document-level sentiment classification on positive and negative movie reviews. ${ }^{6}$

- Yelp Polarity (Yelp): Document-level sentiment classification on positive and negative reviews (Zhang, Zhao, and LeCun 2015). Reviews with a rating of 1 and 2 are labeled negative and 4 and 5 positive.

\section{Textual Entailment}

- SNLI: A dataset of 570K sentence pairs derived from image captions. The task is to judge the relationship between two sentences: whether the second sentence can be derived from entailment, contradiction, or neutral relationship with the first sentence (Bowman et al. 2015).

\footnotetext{
${ }^{5}$ https://www.kaggle.com/c/fake-news/data

${ }^{6}$ https://datasets.imdbws.com/
} 
- MultiNLI: A multi-genre entailment dataset with a coverage of transcribed speech, popular fiction, and government reports (Williams, Nangia, and Bowman 2017). Compared to SNLI, it contains more linguistic complexity with various written and spoken English text.

\section{Attacking Target Models}

For each dataset, we train three state-of-the-art models on the training set, and achieved test set accuracy scores similar to the original implementation, as shown in Table 2. We then generate adversarial examples which are semantically similar to the test set to attack the trained models and make them generate different results.

\begin{tabular}{rccc}
\hline & WordCNN & WordLSTM & BERT \\
\hline AG & 92.5 & 93.1 & 94.6 \\
Fake & 99.9 & 99.9 & 99.9 \\
MR & 79.9 & 82.2 & 85.8 \\
IMDB & 89.7 & 91.2 & 92.2 \\
Yelp & 95.2 & 96.6 & 96.1 \\
\hline & InferSent & ESIM & BERT \\
\hline SNLI & 84.6 & 88.0 & 90.7 \\
MultiNLI & $71.1 / 71.5$ & $76.9 / 76.5$ & $83.9 / 84.1$ \\
\hline
\end{tabular}

Table 2: Original accuracy of target models on standard test sets.

On the sentence classification task, we target at three models: word-based convolutional neural network (WordCNN) (Kim 2014), word-based long-short term memory (WordLSTM) (Hochreiter and Schmidhuber 1997), and the state-ofthe-art Bidirectional Encoder Representations from Transformers (BERT) (Devlin et al. 2018).

For the WordCNN model, we used three window sizes of 3,4 , and 5 , and 100 filters for each window size with dropout of 0.3. For the WordLSTM, we used a 1-layer bidirectional LSTM with 150 hidden units and a dropout of 0.3 . For both models, we used the 200 dimensional Glove word embeddings pre-trained on 6B tokens from Wikipedia and Gigawords (Pennington, Socher, and Manning 2014). We used the 12-layer BERT model with 768 hidden units and 12 heads, with $110 \mathrm{M}$ parameters, which is called the baseuncased version. ${ }^{7}$

We also implemented three target models on the textual entailment task: standard InferSent ${ }^{8}$ (Conneau et al. 2017), $\operatorname{ESIM}^{9}$ (Chen et al. 2016), and fine-tuned BERT.

\section{Setup of Automatic Evaluation}

We first report the accuracy of the target models on the original test samples before attack as the original accuracy. Then we measure the accuracy of the target models against the adversarial samples crafted from the test samples, denoted as after-attack accuracy. By comparing these two accuracy scores, we can evaluate how successful the attack is, - the

\footnotetext{
${ }^{7}$ https://github.com/huggingface/pytorch-pretrained-BERT

${ }^{8} \mathrm{https}$ ://github.com/facebookresearch/InferSent

${ }^{9}$ https://github.com/coetaur0/ESIM
}

larger gap between the original and after-attack accuracy signals the more successful our attack is. Apart from these accuracies, we also report the perturbed word percentage as the ratio of the number of perturbed words to the text length. Furthermore, we apply USE ${ }^{10}$ to measure the semantic similarity between the original and adversarial texts. These two metrics, the perturbed words percentage and the semantic similarity score, together evaluate how semantically similar the original and adversarial texts are. We finally report the number of queries the attack system made to the target model and fetches the output probability scores. This metric can reveal the efficiency of the attack model.

\section{Setup of Human Evaluation}

We conduct human evaluation on three criteria: semantic similarity, grammaticality, and classification accuracy. We randomly select 100 test sentences of each task to generate adversarial examples, one targeting WordLSTM on MR dataset and another targeting BERT on SNLI. We first shuffled a mix of original and adversarial texts and asked human judges to rate the grammaticality of them on a Likert scale of $1-5$, similar to the practice of (Gagnon-Marchand et al. 2018). Next, we evaluate the classification consistency by asking humans to classify each example in the shuffled mix of the original and adversarial sentences and then calculate the consistency rate of both classification results. Lastly, we evaluated the semantic similarity of the original and adversarial sentences by asking humans to judge whether the generated adversarial sentence is similar, ambiguous, or dissimilar to the source sentence. Each task is completed by two independent human judges who are native English speakers. The volunteers have university-level education backgrounds and passed a test batch before they started annotation.

\section{Results}

\section{Automatic Evaluation}

The main results of black-box attacks in terms of automatic evaluation on five text classification and two textual entailment tasks are summarized in Table 3 and 4, respectively. Overall, as can be seen from our results, TEXTFOOLER achieves a high success rate when attacking with a limited number of modifications on both tasks. No matter how long the text sequence is, and no matter how accurate the target model is, TEXTFOOLER can always reduce the accuracy from the state-of-the-art values to below 15\% (except on the Fake dataset) with less than $20 \%$ word perturbation ratio (except the AG dataset under the BERT target model). For instance, it only perturbs $5.1 \%$ of the words on average when reducing the accuracy from $89.8 \%$ to only $0.3 \%$ on the IMDB dataset against the WordLSTM model. Notably, our attack system makes the WordCNN model on the IMDB dataset totally wrong (reaching the accuracy of $0 \%$ ) with only $3.5 \%$ word perturbation rate. In the IMDB dataset which has an average length of 215 words, the system only perturbed 10 words or fewer per sample to conduct successful attacks. This means that our attack system can success-

\footnotetext{
${ }^{10} \mathrm{https}: / /$ tfhub.dev/google/ universal-sentence-encoder
} 


\begin{tabular}{|c|c|c|c|c|c|c|c|c|c|c|c|c|c|c|c|}
\hline & \multicolumn{5}{|c|}{ WordCNN } & \multicolumn{5}{|c|}{ WordLSTM } & \multicolumn{5}{|c|}{ BERT } \\
\hline & MR & IMDB & Yelp & AG & Fake & MR & IMDB & Yelp & AG & Fake & MR & IMDB & Yelp & AG & Fake \\
\hline Original Accuracy & 78.0 & 89.2 & 93.8 & 91.5 & 96.7 & 80.7 & 89.8 & 96.0 & 91.3 & 94.0 & 86.0 & 90.9 & 97.0 & 94.2 & 97.8 \\
\hline After-Attack Accuracy & 2.8 & 0.0 & 1.1 & 1.5 & 15.9 & 3.1 & 0.3 & 2.1 & 3.8 & 16.4 & 11.5 & 13.6 & 6.6 & 12.5 & 19.3 \\
\hline$\%$ Perturbed Words & 14.3 & 3.5 & 8.3 & 15.2 & 11.0 & 14.9 & 5.1 & 10.6 & 18.6 & 10.1 & 16.7 & 6.1 & 13.9 & 22.0 & 11.7 \\
\hline Semantic Similarity & 0.68 & 0.89 & 0.82 & 0.76 & 0.82 & 0.67 & 0.87 & 0.79 & 0.63 & 0.80 & 0.65 & 0.86 & 0.74 & 0.57 & 0.76 \\
\hline Query Number & 123 & 524 & 487 & 228 & 3367 & 126 & 666 & 629 & 273 & 3343 & 166 & 1134 & 827 & 357 & 4403 \\
\hline Average Text Length & 20 & 215 & 152 & 43 & 885 & 20 & 215 & 152 & 43 & 885 & 20 & 215 & 152 & 43 & 885 \\
\hline
\end{tabular}

Table 3: Automatic evaluation results of the attack system on text classification datasets, including the original model prediction accuracy before being attacked ("Original Accuracy"), the model accuracy after the adversarial attack ("After-Attack Accuracy"), the percentage of perturbed words with respect to the original sentence length ("\% Perturbed Words"), and the semantic similarity between original and adversarial samples ("Semantic Similarity").

\begin{tabular}{lcc|cc|cc}
\hline & \multicolumn{2}{c|}{$\begin{array}{l}\text { InferSent } \\
\text { SultiNLI (m/mm) }\end{array}$} & SNLI & $\begin{array}{c}\text { ESIM } \\
\text { MultiNLI (m/mm) }\end{array}$ & SNLI & $\begin{array}{c}\text { BERT } \\
\text { MultiNLI (m/mm) }\end{array}$ \\
\hline Original Accuracy & 84.3 & $70.9 / 69.6$ & 86.5 & $77.6 / 75.8$ & 89.4 & $85.1 / 82.1$ \\
After-Attack Accuracy & 3.5 & $6.7 / 6.9$ & 5.1 & $7.7 / 7.3$ & 4.0 & $9.6 / 8.3$ \\
\% Perturbed Words & 18.0 & $13.8 / 14.6$ & 18.1 & $14.5 / 14.6$ & 18.5 & $15.2 / 14.6$ \\
Semantic Similarity & 0.50 & $0.61 / 0.59$ & 0.47 & $0.59 / 0.59$ & 0.45 & $0.57 / 0.58$ \\
Query Number & 57 & $70 / 83$ & 58 & $72 / 87$ & 60 & $78 / 86$ \\
\hline Average Text Length & 8 & $11 / 12$ & 8 & $11 / 12$ & 8 & $11 / 12$ \\
\hline
\end{tabular}

Table 4: Automatic evaluation results of the attack system on textual entailment datasets. "m" means matched, and "mm" means mismatched, which are the two variants of the MultiNLI development set.

fully mislead the classifiers into assigning wrong predictions via subtle manipulation.

Even for BERT, which has achieved seemingly "robust" performance compared with the non-pretrained models such as WordLSTM and WordCNN, our attack model can still reduce its prediction accuracy by about 5-7 times on the classification task (e.g., from $95.6 \%$ to $6.8 \%$ for Yelp dataset) and about 9-22 times on the NLI task (e.g., from $89.4 \%$ to $4.0 \%$ for SNLI dataset), which is unprecedented. Our curated adversarial examples can contribute to the study of the interpretability of the BERT model (Feng et al. 2018).

Another two observations can be drawn from Table 3 and 4. (1) Models with higher original accuracy is, in general, more difficult to be attacked. For instance, the after-attack accuracy and perturbed word ratio are both higher for the BERT model compared with WordCNN on all datasets. (2) The after-attack accuracy of the Fake dataset is much higher than all other classification datasets for all three target models. We found in experiments that it is easy for the attack system to convert a real news to a fake one, whereas the reverse process is much harder, which is in line with intuition.

Comparing the semantic similarity scores and the perturbed word ratios in both Table 3 and 4, we find that the two results have a high positive correlation. Empirically, when the text length is longer than 10 words, the semantic similarity measurement becomes more stable. Since the average text lengths of text classification datasets are all above 20 words and those of textual entailment datasets are around or below 10 words, we need to treat the semantic similarity scores of these two tasks individually. Therefore, we performed a linear regression analysis between the word perturbation ratio and semantic similarity for each task and

\begin{tabular}{llcc}
\hline Dataset & Model & Success Rate & \% Perturbed Words \\
\hline \multirow{2}{*}{ IMDB } & (Li et al. 2018) & 86.7 & 6.9 \\
& (Alzantot et al. 2018) & 97.0 & 14.7 \\
& Ours & $\mathbf{9 9 . 7}$ & $\mathbf{5 . 1}$ \\
\hline \multirow{2}{*}{ SNLI } & (Alzantot et al. 2018) & 70.0 & 23.0 \\
& Ours & $\mathbf{9 5 . 8}$ & $\mathbf{1 8 . 0}$ \\
\hline \multirow{2}{*}{ Yelp } & (Kuleshov et al. 2018) & 74.8 & - \\
& Ours & $\mathbf{9 7 . 8}$ & $\mathbf{1 0 . 6}$ \\
\hline
\end{tabular}

Table 5: Comparison of our attack system against other published systems. The target model for IMDB and Yelp is LSTM and SNLI is InferSent.

obtained r-squared values of 0.94 and 0.97 for text classification and textual entailment tasks, respectively. Such high values of $\mathrm{r}$-squared reveal that our proposed semantic similarity has high correlation (negative) with the perturbed words ratio, which can both be good automatic measurements to evaluate the degree of alterations of original text.

We include the average text length of each dataset in the last row of Table 3 and 4 so that it can be conveniently compared against the query number. The query number is almost linear to the text lengthm, with a ratio in $(2,8)$. Longer text correlates with a smaller ratio, which validates the efficiency of TEXTFOOLER.

Benchmark Comparison We compared TEXTFoOLER with the previous state-of-the-art adversarial attack systems against the same target model and dataset. Our baselines include ( $\mathrm{Li}$ et al. 2018) that generates misspelled words by character- and word-level perturbation, (Alzantot et al. 2018) that iterates through every word in the sentence and find its perturbation, and (Kuleshov et al. 2018) that uses 


\begin{tabular}{|c|c|}
\hline \multicolumn{2}{|r|}{ Movie Review (Positive (POS) $\leftrightarrow$ Negative (NEG)) } \\
\hline Original (Label: NEG) & The characters, cast in impossibly contrived situations, are totally estranged from reality. \\
\hline Attack (Label: POS) & The characters, cast in impossibly engineered circumstances, are fully estranged from reality. \\
\hline Original (Label: POS) & $\begin{array}{l}\text { It cuts to the knot of what it actually means to face your scares, and to ride the overwhelming } \\
\text { metaphorical wave that life wherever it takes you. }\end{array}$ \\
\hline \multirow[t]{2}{*}{ Attack (Label: NEG) } & $\begin{array}{l}\text { It cuts to the core of what it actually means to face your fears, and to ride the big metaphorical } \\
\text { wave that life wherever it takes you. }\end{array}$ \\
\hline & SNLI (Entailment (ENT), Neutral (NEU), Contradiction (CON)) \\
\hline Premise & Two small boys in blue soccer uniforms use a wooden set of steps to wash their hands. \\
\hline Original (Label: CON) & The boys are in band uniforms. \\
\hline $\begin{array}{l}\text { Adversary (Label: } \\
\text { ENT) }\end{array}$ & The boys are in band garment. \\
\hline Premise & A child with wet hair is holding a butterfly decorated beach ball. \\
\hline Original (Label: NEU) & The child is at the beach. \\
\hline $\begin{array}{l}\text { Adversary (Label: } \\
\text { ENT) }\end{array}$ & The youngster is at the shore. \\
\hline
\end{tabular}

Table 6: Examples of original and adversarial sentences from MR (WordLSTM) and SNLI (BERT) datasets.

\begin{tabular}{lcc}
\hline Source Text & $\begin{array}{c}\text { MR } \\
\text { (WordLSTM) }\end{array}$ & $\begin{array}{c}\text { SNLI } \\
\text { (BERT) }\end{array}$ \\
\hline Original & 4.22 & 4.50 \\
Adversarial & 4.01 & 4.27 \\
\hline
\end{tabular}

Table 7: Grammaticality of original and adversarial examples for MR (WordLSTM) and SNLI (BERT) on $1-5$ scale.

word replacement by greedy heuristics. From the results in Table 5, we can see that our system beats the previous stateof-the-art models by both the attack success rate (calculated by dividing the number of wrong predictions by the total number of adversarial examples) and perturbed word ratio.

\section{Human Evaluation}

We sampled 100 adversarial examples on the MR dataset with the WordLSTM and 100 examples on SNLI with BERT. We verified the quality of our examples via three experiments. First, we ask human judges to give a grammaticality score of a shuffled mix of original and adversarial text.

As shown in Table 7, the grammaticality of the adversarial text are close to the original text on both datasets. By sensibly substituting synonyms, TEXTFOOLER generates smooth outputs such as "the big metaphorical wave" in Table 6.

We then asked the human raters to assign classification labels to a shuffled set of original and adversarial samples. The overall agreement between the labels of the original sentence and the adversarial sentence is relatively high, with $92 \%$ on MR and $85 \%$ on SNLI. Though our adversarial examples are not perfect in every case, this shows that majorities of adversarial sentences have the same attribute as the original sentences from humans' perspective. Table 6 shows typical examples of sentences with almost the same meanings that result in contradictory classifications by the target model.

Lastly, we asked the judges to decide whether each adversarial sample retains the meaning of the original sentence. They need to decide whether the synthesized adversarial ex- ample is similar, ambiguous, or dissimilar to the provided original sentence. We regard similar as 1 , ambiguous as 0.5 , and dissimilar as 0 , and obtained sentence similarity scores of 0.91 on MR and 0.86 on SNLI, which shows the perceived difference between original and adversarial text is small.

\section{Discussion}

\section{Ablation Study}

Word Importance Ranking To validate the effectiveness of Step 1 in Algorithm 1, i.e., the word importance ranking, we remove this step and instead randomly select the words in text to perturb. We keep the perturbed word ratio and Step 2 the same. We use BERT as the target model and test on three datasets: MR, AG, and SNLI. The results are summarized in Table 8. After removing Step 1 and instead randomly selecting the words to perturb, the after-attack accuracy increases by more than $45 \%$ on all three datasets, which reveals that the attack becomes ineffective without the word importance ranking step. The word importance ranking process is crucial to the algorithm in that it can accurately and efficiently locate the words which cast the most significant effect on the predictions of the target model. This strategy can also reduce the number of perturbed words so as to maintain the semantic similarity as much as possible.

\begin{tabular}{lccc}
\hline & MR & AG & SNLI \\
\hline \% Perturbed Words & 16.7 & 22.0 & 18.5 \\
Original Accuracy & 86.0 & 94.2 & 89.4 \\
After-Attack Accuracy & 11.5 & 12.5 & 4.0 \\
\hline After-Attack Accuracy (Random) & $\mathbf{6 8 . 3}$ & $\mathbf{8 0 . 8}$ & $\mathbf{5 9 . 2}$ \\
\hline
\end{tabular}

Table 8: Comparison of the after-attack accuracies before and after removing the word importance ranking of Algorithm 1. For control, Step 2 and the perturbed words ratio are kept the same. BERT model is used as the target model. 
Semantic Similarity Constraint In Step 2 of Algorithm 1 , for every possible word replacement, we check the semantic similarity between the newly generated sample and the original text, and adopt this replacement only when the similarity is above a preset threshold $\epsilon$. We found that this strategy can effectively filter out irrelevant synonyms to the selected word. As we can see from the examples in Table 9, the synonyms extracted by word embeddings are noisy, so directly injecting them into the text as adversarial samples would probably shift the semantic meaning significantly. By applying the sentence-level semantic similarity constraint, we can obtain more related synonyms as good replacements. ${ }^{11}$

\begin{tabular}{ll}
\hline Original & like a south of the border melrose place \\
Adversarial & like a south of the border melrose spot \\
- Sim. & like a south of the border melrose mise \\
\hline Original & their computer animated faces are very expressive \\
Adversarial & their computer animated face are very affective \\
- Sim. & their computer animated faces are very diction \\
\hline
\end{tabular}

Table 9: Qualitative comparison of adversarial attacks with and without the semantic similarity constraint ("-Sim."). We highlight the original word, TextFooler's replacement, and the replacement without semantic constraint.

\begin{tabular}{lrrrr}
\hline & MR & IMDB & SNLI & MNLI(m) \\
\hline After-Attack Accu. & $11.5 / 6.2$ & $13.6 / 11.2$ & $4.0 / 3.6$ & $9.6 / 7.9$ \\
\% Perturbed Words & $16.7 / 14.8$ & $6.1 / 4.0$ & $18.5 / 18.3$ & $15.2 / 14.5$ \\
Query Number & $166 / 131$ & $1134 / 884$ & $60 / 57$ & $78 / 70$ \\
Semantic Similarity & $0.65 / 0.58$ & $0.86 / 0.82$ & $0.45 / 0.44$ & $0.57 / 0.56$ \\
\hline
\end{tabular}

Table 10: Comparison of automatic evaluation metrics with and without the semantic similarity constraint (numbers in the left and right of the symbol " " represent results with and without the constraint, respectively). The target model is BERT-Base.

\section{Transferability}

We examined transferability of adversarial text, that is, whether adversarial samples curated based on one model can also fool another. For this, we collected the adversarial examples from IMDB and SNLI test sets that are wrongly predicted by one target model and then measured the prediction accuracy of them against the other two target models. As we can see from the results in the Table 11, there is a moderate degree of transferability between models, and the transferability is higher in the textual entailment task than in the text classification task. Moreover, the adversarial samples generated based on the model with higher prediction accuracy, i.e. the BERT model here, show higher transferability.

\section{Adversarial Training}

Our work casts insights on how to better improve the original models through these adversarial examples. We con-

\footnotetext{
${ }^{11}$ Please check our arXiv (https://arxiv.org/abs/1907.11932) for a comparison of the automatic evaluation before and after removing the semantic similarity constraint.
}

\begin{tabular}{llccc}
\hline & & WordCNN & WordLSTM & BERT \\
\hline \multirow{3}{*}{ IMDB } & WordCNN & 0.0 & 84.9 & 90.2 \\
& WordLSTM & 74.9 & 0.0 & 87.9 \\
& BERT & 84.1 & 85.1 & 0.0 \\
\hline & & InferSent & ESIM & BERT \\
\hline \multirow{3}{*}{ SNLI } & InferSent & 0.0 & 62.7 & 67.7 \\
& ESIM & 49.4 & 0.0 & 59.3 \\
& BERT & 58.2 & 54.6 & 0.0 \\
\hline
\end{tabular}

Table 11: Transferability of adversarial examples on IMDB and SNLI dataset. Row $i$ and column $j$ is the accuracy of adversaries generated for model $i$ evaluated on model $j$.

ducted a preliminary experiment on adversarial training, by feeding the models both the original data and the adversarial examples (adversarial examples share the same labels as the original counterparts), to see whether the original models can gain more robustness. We collected the adversarial examples curated from the MR and SNLI training sets that fooled BERT and added them to the original training set. We then used the expanded data to train BERT from scratch and attacked this adversarially-trained model. As is seen in the attack results in Table 12, both the after-attack accuracy and perturbed words ratio after adversarial re-training get higher, indicating the greater difficulty to attack. This reveals one of the potency of our attack system,- - we can enhance the robustness of a model to future attacks by training it with the generated adversarial examples.

\begin{tabular}{lcccc}
\hline & \multicolumn{2}{c}{ MR } & \multicolumn{2}{c}{ SNLI } \\
& Af. Acc. & Pert. & Af. Acc. & Pert. \\
\hline Original & 11.5 & 16.7 & 4.0 & 18.5 \\
+ Adv. Training & $\mathbf{1 8 . 7}$ & $\mathbf{2 1 . 0}$ & $\mathbf{8 . 3}$ & $\mathbf{2 0 . 1}$ \\
\hline
\end{tabular}

Table 12: Comparison of the after-attack accuracy ("Af. Acc.") and percentage of perturbed words ("Pert.") of original training ("Original") and adversarial training ("+ Adv. Train") of BERT model on MR and SNLI dataset.

\section{Related Work}

Adversarial attack has been extensively studied in computer vision (Kurakin, Goodfellow, and Bengio 2016a; MoosaviDezfooli et al. 2017). Most works make gradient-based perturbation on continuous input spaces (Szegedy et al. 2013; Goodfellow, Shlens, and Szegedy 2014).

Adversarial attack on discrete data such as text is more challenging. Inspired by the approaches in computer vision, early work in language adversarial attack focus on variations of gradient-based methods. For example, Zhao, Dua, and Singh (2017) transform input data into a latent representation by generative adversarial networks (GANs), and then retrieved adversaries close to the original instance in the latent space.

Other works observed the intractability of GAN-based models on text and the shift in semantics in the latent representations, so heuristic methods such as scrambling, misspelling, or removing words were proposed (Alzantot et 
al. 2018; Li, Monroe, and Jurafsky 2016; Li et al. 2018). Ribeiro, Singh, and Guestrin (2018) automatically craft the semantically equivalent adversarial rules from the machine generated paraphrases using back-translation techniques.

\section{Conclusion}

Overall, we study adversarial attacks against state-of-the-art text classification and textual entailment models under the black-box setting. Extensive experiments demonstrate that the effectiveness of our proposed system, TEXTFOOLER, at generating targeted adversarial texts. Human studies validated that the generated adversarial texts are legible, grammatical, and similar in meaning to the original texts.

\section{Acknowledgements}

We thank Professor Zheng Zhang for insightful discussions, and we appreciate Heather Berlin, Yilun Du, Laura Koemmpel and other helpers for high quality human evaluation.

\section{References}

Alzantot, M.; Sharma, Y.; Elgohary, A.; Ho, B.-J.; Srivastava, M.; and Chang, K.-W. 2018. Generating natural language adversarial examples. arXiv preprint arXiv:1804.07998.

Bowman, S. R.; Angeli, G.; Potts, C.; and Manning, C. D. 2015. A large annotated corpus for learning natural language inference. In Proceedings of the 2015 Conference on Empirical Methods in Natural Language Processing (EMNLP). Association for Computational Linguistics.

Carlini, N., and Wagner, D. 2018. Audio adversarial examples: Targeted attacks on speech-to-text. arXiv preprint arXiv:1801.01944.

Cer, D.; Yang, Y.; Kong, S.-y.; Hua, N.; Limtiaco, N.; John, R. S.; Constant, N.; Guajardo-Cespedes, M.; Yuan, S.; Tar, C.; et al. 2018. Universal sentence encoder. arXiv preprint arXiv:1803.11175.

Chen, Q.; Zhu, X.; Ling, Z.; Wei, S.; Jiang, H.; and Inkpen, D. 2016. Enhanced lstm for natural language inference. arXiv preprint arXiv:1609.06038.

Conneau, A.; Kiela, D.; Schwenk, H.; Barrault, L.; and Bordes, A. 2017. Supervised learning of universal sentence representations from natural language inference data. arXiv preprint arXiv:1705.02364.

Devlin, J.; Chang, M.-W.; Lee, K.; and Toutanova, K. 2018. Bert: Pre-training of deep bidirectional transformers for language understanding. arXiv preprint arXiv:1810.04805.

Feng, S.; Wallace, E.; Grissom, I.; Iyyer, M.; Rodriguez, P.; BoydGraber, J.; et al. 2018. Pathologies of neural models make interpretations difficult. arXiv preprint arXiv:1804.07781.

Gagnon-Marchand, J.; Sadeghi, H.; Haidar, M.; Rezagholizadeh, M.; et al. 2018. Salsa-text: self attentive latent space based adversarial text generation. arXiv preprint arXiv:1809.11155.

Gao, J.; Lanchantin, J.; Soffa, M. L.; and Qi, Y. 2018. Blackbox generation of adversarial text sequences to evade deep learning classifiers. arXiv preprint arXiv:1801.04354.

Goodfellow, I. J.; Shlens, J.; and Szegedy, C. 2014. Explaining and harnessing adversarial examples. arXiv preprint arXiv:1412.6572.

Goodfellow, I.; Shlens, J.; and Szegedy, C. 2015. Explaining and harnessing adversarial examples. In International Conference on Learning Representations.
Hill, F.; Reichart, R.; and Korhonen, A. 2015. Simlex-999: Evaluating semantic models with (genuine) similarity estimation. Computational Linguistics 41(4):665-695.

Hochreiter, S., and Schmidhuber, J. 1997. Long short-term memory. Neural computation 9(8):1735-1780.

Kim, Y. 2014. Convolutional neural networks for sentence classification. arXiv preprint arXiv:1408.5882.

Kuleshov, V.; Thakoor, S.; Lau, T.; and Ermon, S. 2018. Adversarial examples for natural language classification problems.

Kurakin, A.; Goodfellow, I.; and Bengio, S. 2016a. Adversarial examples in the physical world. arXiv preprint arXiv:1607.02533.

Kurakin, A.; Goodfellow, I. J.; and Bengio, S. 2016b. Adversarial machine learning at scale. CoRR abs/1611.01236.

Li, J.; Ji, S.; Du, T.; Li, B.; and Wang, T. 2018. Textbugger: Generating adversarial text against real-world applications. arXiv preprint arXiv:1812.05271.

Li, J.; Monroe, W.; and Jurafsky, D. 2016. Understanding neural networks through representation erasure. arXiv preprint arXiv:1612.08220.

Liang, B.; Li, H.; Su, M.; Bian, P.; Li, X.; and Shi, W. 2017. Deep text classification can be fooled. arXiv preprint arXiv:1704.08006.

Moosavi-Dezfooli, S.-M.; Fawzi, A.; Fawzi, O.; and Frossard, P. 2017. Universal adversarial perturbations. In Proceedings of the IEEE Conference on Computer Vision and Pattern Recognition, $1765-1773$.

Mrkšić, N.; Séaghdha, D. O.; Thomson, B.; Gašić, M.; RojasBarahona, L.; Su, P.-H.; Vandyke, D.; Wen, T.-H.; and Young, S. 2016. Counter-fitting word vectors to linguistic constraints. arXiv preprint arXiv:1603.00892.

Niven, T., and Kao, H.-Y. 2019. Probing neural network comprehension of natural language arguments. arXiv preprint arXiv:1907.07355.

Pang, B., and Lee, L. 2005. Seeing stars: Exploiting class relationships for sentiment categorization with respect to rating scales. In Proceedings of the 43rd annual meeting on association for computational linguistics, 115-124.

Papernot, N.; McDaniel, P.; Goodfellow, I.; Jha, S.; Celik, Z. B.; and Swami, A. 2017. Practical black-box attacks against machine learning. In Proceedings of the 2017 ACM on Asia Conference on Computer and Communications Security, 506-519. ACM.

Pennington, J.; Socher, R.; and Manning, C. 2014. Glove: Global vectors for word representation. In Proceedings of the 2014 conference on empirical methods in natural language processing (EMNLP), 1532-1543.

Ribeiro, M. T.; Singh, S.; and Guestrin, C. 2018. Semantically equivalent adversarial rules for debugging nlp models. In Proceedings of the 56th Annual Meeting of the Association for Computational Linguistics, 856-865.

Szegedy, C.; Zaremba, W.; Sutskever, I.; Bruna, J.; Erhan, D.; Goodfellow, I.; and Fergus, R. 2013. Intriguing properties of neural networks. arXiv preprint arXiv:1312.6199.

Williams, A.; Nangia, N.; and Bowman, S. R. 2017. A broadcoverage challenge corpus for sentence understanding through inference. arXiv preprint arXiv:1704.05426.

Zhang, X.; Zhao, J.; and LeCun, Y. 2015. Character-level convolutional networks for text classification. In Advances in neural information processing systems, 649-657.

Zhao, Z.; Dua, D.; and Singh, S. 2017. Generating natural adversarial examples. arXiv preprint arXiv:1710.11342. 